\title{
Amino acid management of Parkinson's disease: a case study
}

Marty Hinz'

Alvin Stein ${ }^{2}$

Thomas Uncini ${ }^{3}$

'Clinical Research, NeuroResearch Clinics, Inc., Cape Coral, FL, USA;

${ }^{2}$ Stein Orthopedic Associates, Plantation, FL, USA; ${ }^{3}$ DBS Labs, Duluth, MN, USA
Correspondence: Marty Hinz 1008 Dolphin Dr, Cape Coral, FL 33904, USA

$\mathrm{Tel}+\mathrm{I} 2186262220$

Fax +I 2186261638

Email marty@hinzmd.com
This article was published in the following Dove Press journal:

International Journal of General Medicine

25 February 201I

Number of times this article has been viewed

Abstract: An extensive list of side effects and problems are associated with the administration of L-dopa (L-3, 4-dihydroxyphenylalanine) during treatment of Parkinson's disease. These problems can preclude achieving an optimal response with L-dopa treatment.

Purpose: To present a case study outlining a novel approach for the treatment of Parkinson's disease that allows for management of problems associated with L-dopa administration and discusses the scientific basis for this treatment.

Patients and methods: The case study was selected from a database containing 254 Parkinson's patients treated in developing and refining this novel approach to its current state. The spectrum of patients comprising this database range from newly diagnosed, with no previous treatment, to those who were diagnosed more than 20 years before and had virtually exhausted all medical treatment options. Parkinson's disease is associated with depletion of tyrosine hydroxylase, dopamine, serotonin, and norepinephrine. Exacerbating this is the fact that administration of L-dopa may deplete L-tyrosine, L-tryptophan, 5-hydroxytryptophan (5-HTP), serotonin, and sulfur amino acids. The properly balanced administration of L-dopa in conjunction with 5-HTP, L-tyrosine, L-cysteine, and cofactors under the guidance of organic cation transporter functional status determination (herein referred to as "OCT assay interpretation") of urinary serotonin and dopamine, is at the heart of this novel treatment protocol.

Results: When 5-HTP and L-dopa are administered in proper balance along with L-tyrosine, L-cysteine, and cofactors under the guidance of OCT assay interpretation, the long list of problems that can interfere with optimum administration of L-dopa becomes controllable and manageable or does not occur at all. Patient treatment then becomes more effective by allowing the implementation of the optimal dosing levels of L-dopa needed for the relief of symptoms without the dosing value barriers imposed by side effects and adverse reactions seen in the past.

Keywords: Parkinson's, Parkinsonism, Parkinson's disease, L-dopa, 5-HTP

\section{Introduction}

There is a need to effectively control and manage the problems associated with L-dopa treatment in every Parkinson's patient allowing full access to the L-dopa dosing level needed by each Parkinson's patient in order to achieve optimal relief of symptoms. ${ }^{1,2}$

This novel approach allows for the full access to the required L-dopa dosing values through minimization or elimination of the undesirable L-dopa side effects. This is the first attempt to formally document some of the results seen and the experience gained in refining this novel protocol as applied to Parkinson's disease.

Based on clinical experience gained with this novel protocol, it is asserted that virtually all of the problems encountered in the administration of L-dopa for Parkinson's 
disease are caused by the improper management of systems impacted by L-dopa and/or the concomitant use of carbidopa with L-dopa.

This case study was selected as the focal point for a discussion of a novel Parkinson's disease treatment protocol. The case was not selected due to extraordinary results; it was selected as an illustration of typical results seen with this protocol. The purpose of this paper is to outline the basic protocol as a reference point for future prospective studies.

The patient is a Vietnam veteran working as a computer specialist who at the age of 55 years began to experience tremor in the left upper extremity, which led to the diagnosis of Parkinson's disease. The patient was referred to the practice of one of the authors of this paper for intravenous (IV) glutathione treatment by a prominent neurologist. The patient had exhausted virtually all nonsurgical medical approaches in the treatment of his Parkinson's disease. His neurologist advised him that the only remaining option available in the United States was deep brain stimulation. This option was not agreeable to the patient.

This paper is based on research started in 1997 that has consistently focused on the study, interactions, and applications of serotonin, dopamine, and their amino acid precursors. The hypothesis of this writing is that the majority of side effects and problems observed during treatment of Parkinson's disease with L-dopa are caused by mismanagement of the amino acid precursors and systems affected by L-dopa.

Side effects and/or adverse reactions tend to be the dose-limiting events associated with administration of only L-dopa. These include but are not limited to nausea, involuntary movements, and psychiatric problems. ${ }^{1,2}$ In order to address these L-dopa problems, medications that potentiate L-dopa and/or dopamine are conventionally administered. These include decarboxylase inhibitors (carbidopa), ${ }^{3}$ dopamine agonists, ${ }^{4}$ glutamate (N-methyl-D-aspartic acid [NMDA])-blocking drugs, ${ }^{5}$ anticholinergics, ${ }^{6}$ MAO-B inhibitors, ${ }^{7}$ and COMT inhibitors. ${ }^{8}$ All of these drugs are second-line support drugs in comparison to the correctional action and potential of L-dopa. ${ }^{9}$

This novel approach for the treatment of Parkinson's disease is dependent upon the administration of L-dopa in adequate amounts to control symptoms through minimization of side effects and adverse reactions by establishing a proper balance between the dopamine and serotonin systems with the concomitant use of 5-hydroxytyrptophan (5-HTP), L-tyrosine, and a sulfur amino acid under the guidance of organic cation transporter (OCT) assay interpretation. ${ }^{10-14}$
The primary symptoms of Parkinson's disease are the result of degeneration of the post-synaptic neurons of the substantia nigra. The very process of Parkinson's disease is associated with depletion of dopamine (DA), tyrosine hydroxylase $(\mathrm{TH})$, norepinephrine (NE), and serotonin (5-HT). ${ }^{15-17}$ Parkinson's disease depletion of these systems is compounded by further depletion with L-dopa. As noted in the literature, administration of only L-dopa or improperly balanced L-dopa further depletes:

- L-tyrosine e $^{13,18,19}$

- $\operatorname{serotonin}^{10,14,18,20-25}$

- L-tryptophan ${ }^{18}$

- sulfur amino acids (glutathione and S-adenosylmethionine $)^{10,13,14,26-28}$

- epinephrine $^{29}$

\section{L-dopa depletion of L-tyrosine}

Patients with Parkinsonism suffer from low levels of tyrosine hydroxylase prior to treatment. ${ }^{15,17}$ The depressed levels of tyrosine hydroxylase inhibit conversion of L-tyrosine to L-dopa. It is known that L-dopa depletes L-tyrosine. ${ }^{13,18,19}$ The interaction of L-tyrosine and L-dopa is covered in the discussion section of this manuscript. Other considerations of L-tyrosine depletion by L-DOPA that need to be explored are the impact on other systems where L-tyrosine acts as a precursor, such as with thyroid hormones.

\section{L-dopa depletion of serotonin}

Patients with Parkinsonism suffer from inadequate levels of serotonin as a result of the disease state. ${ }^{15,16} \mathrm{On}$ average, Parkinson's disease patients have a 50\% depletion of serotonin prior to starting treatment. ${ }^{16}$ Significant dosing values of L-dopa induce the competitive inhibition state leading to further serotonin depletion through processes relating to interaction of serotonin and dopamine in synthesis, transport, and metabolism. ${ }^{10,14,18,20-25}$ Definitive steps need to be taken during administration of L-dopa to keep serotonin in proper balance with dopamine. ${ }^{10,13,14}$

In the competitive inhibition state the serotonin and catecholamine systems function as one single system (herein referred to as the "serotonin-dopamine system"). ${ }^{10-14}$ While in the competitive inhibition state changes to one component of the serotonin-dopamine system will effect changes to all components of both systems. ${ }^{11,14}$ The solution is concomitant administration of 5-HTP along with L-dopa to prevent the serotonin depletion. ${ }^{10,13,14}$ 


\section{L-dopa depletion of sulfur amino acids}

It is known that administration of L-dopa depletes sulfur amino acids. ${ }^{10,13,14,26-28}$ The implications of this are extensive. Glutathione is a sulfur amino acid and a powerful antioxidant that neutralizes neurotoxins that may cause Parkinson's disease and other neurotoxic events in the body. ${ }^{25}$ Implications of sulfur amino acid depletion relating to Parkinson's disease include but are not limited to the depletion of:

- glutathione leading to progression of Parkinson's disease if the neurotoxic agents that are a component of the etiology are still present and being absorbed into the system ${ }^{25}$

- the enzymes required to synthesize L-tyrosine to L-dopa ${ }^{13,17,19}$

- s-adenosyl-methionine, the body's one carbon methyl donor ${ }^{10,13,14,26-28}$

- epinephrine $^{29}$

\section{Carbidopa in treatment}

Carbidopa is a general decarboxylase inhibitor. It inhibits L-aromatic amino acid decarboxylase (AAAD), the enzyme that catalyzes synthesis of both serotonin and dopamine from 5-HTP and L-dopa, respectively. Carbidopa does not cross the bloodbrain barrier. It exerts its actions peripherally. In Parkinson's disease it is administered to decrease peripheral conversion of L-dopa to dopamine. This results in the need for a lesser dose of L-dopa peripherally while still giving the higher level in the CNS with fewer side effects, especially nausea. Carbidopa is used to address the side effects seen when improperly balanced L-dopa is administered; there is no direct therapeutic value of carbidopa in treatment of Parkinson's disease. ${ }^{30}$

Carbidopa's inhibition of AAAD potentiates peripheral serotonin, dopamine, norepinephrine, and epinephrine depletion as synthesis by AAAD is compromised. Norepinephrine and acetylcholine regulate autonomic nervous system function. The administration of carbidopa with L-dopa is replete with peripheral autonomic dysfunction problems that often develop. ${ }^{30}$

Administration of carbidopa/L-dopa preparations leads to a "double depletion" of peripheral serotonin. One cause of depletion is carbidopa inhibition of AAAD; the other cause is improperly balanced administration of L-dopa which decreases peripheral serotonin synthesis and transport through competitive inhibition along with increasing the metabolism of serotonin. ${ }^{10,13,14}$

\section{Materials and methods}

Organic cation transporter functional status determination (herein referred to as "OCT assay interpretation") along with the amino acid dosing values described in this paper were developed by NeuroResearch Clinics, Inc (Duluth, $\mathrm{MN})$. All of the amino acid components, including L-dopa, are available over the counter without a prescription in the United States.

Treatment was initiated with administration of 5-HTP, L-tyrosine, and L-dopa at the dose levels shown in Table 1. In addition to the amino acids noted in Table 1, the following cofactors were administered daily: 1) vitamin C 1,000 mg; 2) calcium citrate $220 \mathrm{mg}$; 3) vitamin B6 $75 \mathrm{mg}$; 4) folate $400 \mathrm{mcg}$; 5) L-lysine $500 \mathrm{mg}$; 6) L-cysteine 4,500 mg; 7) selenium $400 \mathrm{mcg}$.

$\mathrm{L}$-dopa in the form of mucuna pruriens $40 \%$ standardized was used. Peer-reviewed literature suggests that L-dopa from the mucuna pruriens source has more rapid onset of action and a longer time of effectiveness leading to the conclusion that it may be a superior source of L-dopa in treating Parkinson's disease. ${ }^{31}$

\section{OCT assay interpretation overview}

Serotonin and dopamine are found in two states. The endogenous state exists when no amino acid precursors are administered. The competitive inhibition state occurs when significant amounts of the monoamine precursors are simultaneously administered. Proper OCT assay interpretation requires that both serotonin and dopamine be placed in the competitive inhibition state prior to the assay interpretation being performed for the results to be valid.

The scientific basis for OCT assay interpretation is novel having been published in 2009 and 2010. At the heart of this approach is the " 3 -phase model." "10-14,32

The goal of this novel approach is to keep the urinary serotonin in the phase III therapeutic range, no higher than $800 \mu \mathrm{g}$ serotonin per gram of creatinine, through proper manipulation of 5-HTP in combination with L-dopa dosing values under the guidance of OCT assay interpretation. $3,7,8,19,20$

Table I Parkinson's dosing values at protocol initiation. Serotonin and dopamine precursors initially administered. One week after start of the second dosing level, a urine sample was obtained for OCT assay interpretation

\begin{tabular}{|c|c|c|c|}
\hline \multicolumn{4}{|c|}{ mg 5-hydroxytryptophan (5-HTP)/mg L-tyrosine/mg L-dopa } \\
\hline & Morning & Noon & 4 pm \\
\hline Initial visit & $150 / 1,500 / 120$ & - & $150 / 1,500 / 120$ \\
\hline 7 days into treatment & $150 / 1,500 / 360$ & $0 / 0 / 240$ & $150 / 1,500 / 360$ \\
\hline 14 days into treatment & \multicolumn{3}{|c|}{ Initiate organic cation transporter assay } \\
\hline
\end{tabular}


To facilitate replication of results the following data is included. Processing, management, and assay of the urine samples collected for this study were as follows. Urine samples were collected about 6 hours prior to bedtime with 4:00 pm being the most frequent collection time point. The samples were stabilized in $6 \mathrm{~N} \mathrm{HCl}$ to preserve the dopamine and serotonin. The urine samples were collected after a minimum of 1 week during which time the patient was taking a specific daily dosing of amino acid precursors of serotonin and dopamine where no doses were missed. Laboratory studies were conducted by DBS Laboratories under the direction of Thomas Uncini, MD, one of the authors. The assays were performed using commercially available radioimmunoassay kits from Immunol Biological Laboratories, Inc (Minneapolis, MN). Assays were interpreted by Marty Hinz of NeuroResearch Clinics.

\section{Evaluation of treatment results}

The Unified Parkinson's Disease Rating Scale (UPDRS) on presentation into the practice and before the start of the protocol as compared to the results 2 years into the amino acid treatment is seen in Table 2.

\section{Results}

The only portion of the patient's history that seems relevant to the etiological factors involved in the development of the Parkinson's disease was his exposure to Agent Orange during the course of his tour of duty in Vietnam while serving in the military. Agent Orange is a known neurotoxin.

Early in the patient's clinical course the symptoms were mild and untreated. As the symptoms increased in the left upper extremity he was prescribed carbidopa-levodopa. The patient was opposed to taking the carbidopa-levodopa at that time and pursued alternative medicine options. Over the next 18 months, the patient was treated with IV glutathione, amantadine, trihexyphenidyl, primidone, and propranolol.

The amantadine gave no relief. The trihexyphenidyl was given in an attempt to relieve the tremor, but the tremor continued to progress extending into the patient's left

Table 2 Unified Parkinson's disease rating scale (UPDRS) ${ }^{33}$

\begin{tabular}{lll}
\hline & $\begin{array}{l}\text { Start of } \\
\text { treatment }\end{array}$ & $\begin{array}{l}\mathbf{2} \text { years into treatment } \\
\text { with amino acids }\end{array}$ \\
\hline Mentation behavior mood & 9 & 0 \\
Daily activities of living & 16 & 3 \\
Motor examination & 16 & 0 \\
Complications of therapy & $\mathrm{I}$ & 0 \\
Totals & 42 & 3 \\
\hline
\end{tabular}

lower extremity compounded by severe balance and gait issues. Primidone provided some relief from the tremor, but it caused severe nausea requiring discontinuance of the medication. Propranolol provided no relief. IV glutathione was effective in diminishing the tremor, but results lasted only about 30 hours.

The patient was then again prescribed carbidopalevodopa in combination, which was completely ineffective. It was also suggested by that physician that the patient seek out additional IV glutathione treatments nearer to his home as that was the only approach that achieved some relief of symptoms.

In October 2008, the patient presented at the practice of one of the authors of this paper for IV glutathione treatment. The patient was having increasing difficulty with every aspect of life. He was very self-conscious of the constant tremor in his left arm and left leg, which interfered with sleep and sitting comfortably in a chair, and made him feel conspicuous in public. He was having great difficulty working at his job as a computer specialist for a large computer corporation and found it almost impossible to type on a keyboard or to maintain focus during conference calls. He was also experiencing severe anxiety, depression, panic attacks, and insomnia.

On the first visit it was apparent that IV glutathione had not achieved any meaningful or lasting relief of symptoms in the past and that it was another brief band-aid approach to the disease. After obtaining a comprehensive medical history and performing a physical examination the patient was offered the novel treatment approach discussed in this report. The use of comprehensive amino acid support in conjunction with OCT assay interpretation was discussed with the patient and he agreed to the treatment plan.

Within 4 months of initiating treatment the patient experienced dramatic improvement in the tremor in both the upper and lower extremities. He regained coordination in his left hand and was once again able to use the computer keyboard. He resumed his hobby of guitar playing and was able to perform proficiently. His gait and balance were restored. The depression improved significantly. Anxiety was significantly relieved. The patient had lost his fear of going out in public.

In the 2 years since initiation of treatment the patient has continuously maintained the benefit of the treatment, with no surgical intervention as previously recommended by his neurologist and no prescription medications.

There appears to have been some progression of the damage associated with the Parkinson's neurons since 
initiation of treatment, as there has been a need for increasing the dosage of the L-dopa. This has been managed by increasing the L-dopa dosing value while continuing to monitor the balance of the levels with OCT assay interpretation. The reason for asserting that this represented a progression of disease state versus tachyphylaxis of L-dopa, which is commonly seen during treatment of Parkinson's disease, is covered in the Discussion section.

The amino acid dosing that the patient was taking at the initiation of this study, 2 years after starting on the program, is found in Table 3 .

The amino acid dosing values administered in treatment were guided by OCT assay interpretation to assist in proper titration of the levels of L-dopa, L-tyrosine, and 5-HTP. This assured that the patient achieved proper therapeutic levels of amino acids while keeping serotonin and dopamine in the proper balance required for minimization of side effects and adverse reactions while optimizing outcomes. The urinary serotonin and dopamine levels obtained during stabilization of the patient were as shown in Table 4.

At the April 29, 2010 collection date sample of Table 4, the patient was suffering from nausea and on-off effect secondary to serotonin imbalance and inadequate L-tyrosine administration, respectively. The nausea improved by the May 18, 2010 collection date sample. Subsequent to the May 18, 2010 collection date the L-tyrosine was increased incrementally to $10,375 \mathrm{mg}$ per day and the L-dopa was gradually lowered. By mid-June, the nausea and on-off effect became minimal.

\section{Discussion Etiology of the disease}

The literature is clear that L-dopa holds the highest potential for relief of symptoms, but dosing values in many patients are limited by side effects. ${ }^{9}$ With administration of L-dopa, side effects and adverse reactions may be the dose-limiting event that prevents attaining optimal dosing values. The following discussion is aimed at defining the basis for an

Table 3 The current amino acid dosing values that the patient is taking, plus cofactors found in the discussion associated with Table I

\begin{tabular}{ll}
\hline Present daily amino acid dosing values in $\mathbf{m g} /$ day & \\
\hline L-dopa & 14,700 \\
5-HTP & 37.5 \\
L-tyrosine & 10,375 \\
L-cysteine & 4,500 \\
L-tryptophan & 375 \\
\hline
\end{tabular}

effective treatment that controls the problems associated with L-dopa administration.

There are a host of problems encountered in group treatment with L-dopa that have not been displayed in this patient's course of treatment. ${ }^{1,30}$ Future prospective studies under this protocol are indicated to define a more exact incidence of each of these events and their impacts on treatment.

While this is a case study report, the authors of this paper possess data on the amino acid dosing needs of over 254 Parkinson's patients who have been treated in the refinement of this novel approach to its current state. At the core of this approach is the administration of L-dopa, which is approved by the US Food and Drug Administration (FDA) for treatment of Parkinson's disease. Table 5 lists the group dosing ranges of 5-HTP and L-dopa seen during refinement of this novel Parkinson's disease treatment approach with the guidance of OCT assay interpretation.

As noted in Table 5, dosing value needs are highly individualized. Group dosing value needs of L-dopa and 5-HTP to control the symptoms vary in exceptionally large ranges. The L-dopa dosing value found in the "high" column of Table 5 has not been described previously in the literature for the treatment of Parkinson's disease. It appears that adverse reactions have precluded reaching these dosing values in the past.

The chief dose-limiting factor tends to be nausea. ${ }^{2}$ Our experience indicates that the only way a patient can safely and effectively be titrated to the high L-dopa dosing values found in Table 5 is with OCT assay interpretation guiding amino acid dosing values.

In Table 3 of this case study the daily L-dopa dosing value of $14,700 \mathrm{mg}$ per day is large. The authors of this paper were unable to locate previous literature discussing this type of dosing value being routinely available to patients if needed without seeing a problem significant enough to cut back the treatment below the therapeutic threshold. Review of the literature reveals that a significant group of patients develop the gastrointestinal (GI) side effects that limit further increases in L-dopa with dosing values in the 3,000 to 4,000 $\mathrm{mg}$ per day range. Certainly there are Parkinson's patients that need much more than 3,000 to 4,000 $\mathrm{mg}$ per day of L-dopa, but these patients are barred from the optimal treatment benefits of L-dopa as a result of the side effects. ${ }^{1,2}$

The huge dosing variance seen during group treatment of the Parkinson's patients in Table 5 shows that one-size dosing does not fit all. 
Table 4 Serotonin and dopamine values reported in $\mu \mathrm{g}$ of monoamine per $\mathrm{g}$ of creatinine. Amino acid dosing values reported in mg/day

\begin{tabular}{|c|c|c|c|c|c|c|c|}
\hline I & 2 & 3 & 4 & 5 & 6 & 7 & 8 \\
\hline Date & Serotonin & Serotonin phase & Dopamine & Dopamine phase & 5-HTP & L-tyrosine & L-dopa \\
\hline $10 / 23 / 2008$ & I,309.I & 3 & 345.8 & 2 & 200.0 & 1,500 & 300 \\
\hline $10 / 30 / 2008$ & I,882.I & 3 & 341.4 & 2 & 200.0 & 3,000 & 300 \\
\hline I I/6/2008 & $7,506.8$ & 3 & 792.9 & 3 & 300.0 & 3,000 & 450 \\
\hline $11 / 18 / 2008$ & $3,962.9$ & 3 & $1,212.2$ & 3 & 100.0 & 3,000 & 1950 \\
\hline $12 / \mid 1 / 2008$ & $2,192.5$ & 3 & $\mathrm{I}, 364.7$ & 3 & 100.0 & 4,500 & 3600 \\
\hline $1 / 8 / 2009$ & 106.7 & 3 & $1,900.6$ & 3 & 37.5 & 4,500 & 6300 \\
\hline $1 / 22 / 2009$ & 399.9 & 3 & $\mathrm{I}, 170.8$ & 3 & 37.5 & 4,500 & 6300 \\
\hline $2 / 12 / 2009$ & 202.1 & 3 & $\mathrm{I}, 747.7$ & 3 & 37.5 & 4,500 & 8400 \\
\hline $3 / 19 / 2009$ & $\mathrm{I}, 175.8$ & 3 & $\mathrm{I}, 489.9$ & 3 & 100.0 & 4,500 & 15,750 \\
\hline $4 / 13 / 2009$ & 395.2 & 3 & $1,149.9$ & 3 & 100.0 & 4,500 & 12600 \\
\hline $6 / 1 / 2009$ & 386.3 & 3 & $1,8 \mid 4.6$ & 3 & 37.5 & 4,875 & 11,550 \\
\hline $6 / 18 / 2009$ & 130.2 & 3 & I,729.7 & 3 & 37.5 & 4,875 & 12,600 \\
\hline $7 / 28 / 2009$ & 84.9 & 3 & $2,915.7$ & 3 & 37.5 & 4,875 & 12,600 \\
\hline $9 / 8 / 2009$ & 386.3 & 3 & $1,730.0$ & 3 & 37.5 & 3,875 & 12,600 \\
\hline $1 / 7 / 2010$ & 710.2 & 3 & $2,029.8$ & 3 & 75.0 & 5,750 & 14,700 \\
\hline $4 / 29 / 2010$ & $4,848.6$ & 3 & $1,346.0$ & 3 & 212.5 & 7,125 & 13,350 \\
\hline $5 / 18 / 2010$ & 724.9 & 3 & I,505.7 & 3 & 37.5 & 6,375 & 14,700 \\
\hline
\end{tabular}

Notes: I) Date of urine sample collection; 2) urinary serotonin reported; 3) serotonin phase; 4) urinary dopamine reported; 5) dopamine phase; 6) 5-HTP dosing value at time of sample collection; 7) L-tyrosine dosing value at time of sample collection; 8) L-dopa dosing value at time of sample collection.

\section{Management of L-dopa depletion of serotonin}

The serotonin depletion symptoms seen in Parkinson's disease and L-dopa administration fall into three categories: 1) disease symptoms associated with inadequate serotonin levels; 2) side effects and adverse reactions; and 3) tachyphylaxis of L-dopa.

Depression is a prominent disease associated with inadequate serotonin levels in Parkinson's disease patients during treatment with L-dopa. Proof of this is the response to the most selective serotonin reuptake inhibitor, citalopram. ${ }^{34}$ Other monoamine-related disease symptoms resulting from depletion of serotonin by the disease and L-dopa are covered in the following discussion. ${ }^{10-13}$

Simply administering some 5-HTP with L-dopa is not optimal. When serotonin levels that are too high in phase III depletion of dopamine may occur through competitive inhibition. ${ }^{10,14,35,36}$ Nausea associated with the administration of L-dopa is a sign of serotonin and dopamine imbalance. If

Table 5 5-HTP and L-dopa group dosing parameters based on OCT assay interpretation during treatment $\mathrm{N}=7$ 14.5-HTP and L-dopa dosing value range (low-high), mean, and standard deviation

\begin{tabular}{lllll}
\hline & Low & High & Mean & SD \\
\hline 5-HTP & $37.5 \mathrm{mg}$ & $2,100 \mathrm{mg}$ & $300 \mathrm{mg}$ & $330.3 \mathrm{mg}$ \\
L-dopa & $120 \mathrm{mg}$ & $25,230 \mathrm{mg}$ & $1,680 \mathrm{mg}$ & $3,652 \mathrm{mg}$ \\
\hline
\end{tabular}

Note: OCT assay interpretation refers to organic cation transporter functional status determination. serotonin levels are too high or too low, nausea from L-dopa can become a significant problem. When 5-HTP and L-dopa are in proper balance and nausea associated with L-dopa displays, it is much milder and more easily manageable, resolving in a matter of days in most cases. Additional management of residual nausea may be through the implementation of smaller, more frequent L-dopa dosing values. With proper serotonindopamine balance nausea is no longer an L-dopa dose-limiting event for virtually all patients and the use of carbidopa is no longer needed. Since the development of nausea is the result of serotonin levels that are either too high or too low and the high or low status of the serotonin relative to dopamine cannot be distinguished clinically, OCT assay interpretation is indicated to properly clarify and manage the problem.

Even with judicious and generous use of OCT assay interpretation in this case study, this patient developed some transient nausea, but it was not significant enough to avoid maintaining the L-dopa dosing values at the level needed for continued relief of Parkinson's symptoms or the stopping of the L-dopa, and within a matter of days the symptoms resolved. ${ }^{10-14}$

Tachyphylaxis (where a drug stops working) of L-dopa is associated with depletion of serotonin by L-dopa. When serotonin levels become too depleted, any beneficial effects of L-dopa will not be observed regardless of how high the L-dopa dosing values are raised. When tachyphylaxis occurs, the typical response is to increase the daily L-dopa dosing value, which only further depletes serotonin. As treatment 
progresses, with further increases of the L-dopa dosing values, the time between tachyphylaxis events decreases and the patient's L-dopa dosing values spiral ever higher. The indicated approach is OCT assay interpretation in order to balance serotonin and its precursors with the L-dopa and dopamine, thereby managing the problem effectively. ${ }^{10-14}$

The results section noted a decrease in effectiveness in the L-dopa in this patient in early 2009. In the results section it was asserted, "There appears to have been some progression of the damage associated with the Parkinson's neurons since the initiation of treatment, as there has been a need for increasing the dosage of the L-dopa. This has been managed by increasing the L-dopa dosing value while continuing to monitor the balance of the levels with OCT assay interpretation." The decreased effectiveness of L-dopa is primarily due to one of three events: 1) lack of patient compliance; 2) serotonin depletion leading to L-dopa tachyphylaxis; or 3) further neuronal damage causing progression of the disease. The patient's medication journal ruled out concerns in the area of compliance. OCT assay interpretation revealed that the urinary serotonin was in phase III and in the desired range, ruling out serotonin depletion concerns associated with L-dopa tachyphylaxis. By exclusion, further progression of disease in the form of progression of neuronal damage had occurred. The three primary causes of decreased response to prescribing L-dopa noted in this paragraph need to be fully appreciated and implemented since the treatment approach to the perceived decrease in efficacy of L-dopa is very different.

\section{Management of dopamine fluctuations with L-tyrosine}

As noted in previous writings by two of the authors of this paper, urinary dopamine levels fluctuate on urinary assay significantly if proper levels of L-tyrosine are not co-administered with L-dopa in the competitive inhibition state. ${ }^{37}$ This fluctuation in the competitive inhibition state is a direct reflection of OCT2 activity of the basolateral monoamine transporters of the proximal convoluted tubule cells of the kidneys. ${ }^{11,38}$ Research experience leading up to this writing has shown that patients taking L-dopa required minimum administration of 5,000 to 6,000 mg per day of L-tyrosine to prevent significant urinary dopamine fluctuations. ${ }^{10,13}$ It is a novel finding of this research that L-tyrosine depletion and dopamine fluctuations are associated with the on-off effect. The patient in this study began to experience on-off effect. Instead of increasing the L-dopa dosing, the L-tyrosine dosing values were increased as noted in Table 3. The maximum required L-tyrosine dosing value encountered for control of on-off effect in all patients studied leading up to the writing of this paper was $20,000 \mathrm{mg}$ per day.

\section{Management of sulfur amino acid depletion by L-dopa}

The sulfur amino acid L-cysteine was selected for use due to its role in synthesis of enzymes that catalyze monoamine synthesis. Theoretically, from the standpoint of enzymes that catalyze monoamine synthesis, any sulfur amino acid, with the exception of $\mathrm{N}$-acetyl-cysteine and glutathione, may serve as a sulfur donor in enzyme synthesis. From a database developed by one of the authors of this article containing over 1.931 million patient-days of amino acid treatment in patients not suffering from Parkinson's disease, objective results revealed optimal L-cysteine dosing was 4,500 $\mathrm{mg}$ per day. No objective changes were observed with the daily dosing values of L-cysteine at or below 2,250 mg per day and no additional response was seen in dosing values greater than 4,500 mg per day. ${ }^{10,13,14}$

Administration of proper levels of sulfur amino acids prevents depletion of all of the following: glutathione; the enzymes that catalyze amino acid precursors into monoamines; S-adenosylmethionine; and epinephrine. ${ }^{10,13,14}$

It is asserted that metabolism of toxins utilizes a large amount of sulfur amino acids in the form of glutathione each day. Administration of IV glutathione is analogous to temporarily plugging a hole in a bucket leaking sulfur amino acids. The effect of IV glutathione is a temporary band-aid approach, with sulfur amino acid levels returning to the previous state and a relapse of symptoms within one to two days of administration. A superior approach is the daily administration of proper levels of sulfur amino acids from the start of treatment so that sulfur amino acid depletion does not have to be further addressed. ${ }^{10,13,14}$

\section{Management of paradoxical amino acid reactions}

Most Parkinson's disease patients treated under this novel approach do not achieve gradual relief of symptoms as the L-dopa dosing values are increased. Symptom cessation tends to be abrupt, analogous to turning a light switch from off to on. ${ }^{10,13,14}$

Paradoxical reactions with concomitant administration of serotonin and dopamine amino acid precursors occur in approximately $5 \%$ of patients. A paradoxical reaction is 
defined as an outcome to treatment that is the opposite of what is expected. In the Parkinson's disease patients being treated with balanced amino acid precursors, the most common paradoxical reactions are agitation and confusion, although any disease process related to monoamine diseases may be exacerbated such as depression, insomnia, or anxiety. With most Parkinson's disease patients, paradoxical reactions occur after many weeks or months when the L-dopa dosing value is on the threshold needed for control of Parkinson's disease symptoms late in treatment. . $^{10,13,14}$

Achieving the results noted in this case study is dependent upon proper management of any paradoxical reactions that may develop and being able to differentiate a paradoxical reaction from a side effect or adverse reaction. Proper management of paradoxical reactions in the Parkinson's patient is to adequately increase the L-dopa dosing value. With a proper increase, the paradoxical reaction will resolve in 1 to 2 days. Physicians who are not properly oriented to the management of paradoxical reactions may tend to inappropriately decrease the L-dopa dosing when a paradoxical reaction displays, then increase the L-dopa dosing slowly. This approach only leads to the patient being exposed to the L-dopa dosing range that induced the paradoxical reaction for a prolonged period of time and may lead to failure when symptoms of a paradoxical reaction are observed for a prolonged period of time causing the L-dopa dosing value to be decreased further or stopped. ${ }^{10,13,14}$

\section{Categorizing symptoms associated with carbidopa/L-dopa administration}

The FDA-approved prescribing information for carbidopa/ L-dopa preparations was reviewed and a list of side effects, adverse reactions, and problems associated with administration was generated. ${ }^{34}$ Each side effect was then placed in one or more of the general categories listed below by the authors of this paper. While the listing of each side effect may be open to further discussion, these are the categories that have evolved in this research project since 2001. The six categories of carbidopa/L-dopa side effects are as follows:

Category 1: Problems caused by depletion of serotonin by L-dopa: Tachyphylaxis (the L-dopa stops working).

Category 2: Problems caused by imbalance of serotonin and dopamine: Nausea, vomiting, anorexia, weight loss, decreased mental acuity, depression, psychotic episodes including delusions, euphoria, pathologic gambling, impulse control, confusion, dream abnormalities including nightmares, anxiety, disorientation, dementia, nervousness, insomnia, sleep disorders, hallucinations and paranoid ideation, somnolence, memory impairment, and increased libido.

Category 3: Problems caused by dopamine fluctuations due to inadequate tyrosine levels: On-off effect, motor fluctuations, dopamine fluctuations, implicated as an etiology of dyskinesia.

Category 4: Problems caused by depletion of sulfur amino acids by L-dopa: Bradykenesia (epinephrine depletion implicated), akinesia, dyskinesia, dystonia, chorea, extrapyramidal side effects, fatigue, abnormal involuntary movements, and depletion of glutathione potentiating further dopamine neuron damage by neurotoxins.

Category 5: Problems caused by paradoxical amino acid reactions: Confusion, dizziness, headache, palpitations, dyspnea, anxiety, agitation, increased tremor, faintness, exacerbation of any disease related to the monoamine (serotonin, dopamine, norepinephrine, and epinephrine) neurotransmitters, and exacerbation of any central disease process associated with the serotonin and catecholamine systems.

Category 6: Peripheral problems caused by peripheral depletion of serotonin and catecholamines by carbidopa: Glossitis, leg pain, ataxia, falling, gait abnormalities, blepharospasm (which may be taken as an early sign of excess dosage), trismus, increased tremor, numbness, muscle twitching, peripheral neuropathy, myocardial infarction, flushing, oculogyric crises, diplopia, blurred vision, dilated pupils, urinary retention, urinary incontinence, dark urine, hoarseness, malaise, hot flashes, sense of stimulation, dyspepsia, constipation, palpitation, fatigue, upper respiratory infection, bruxism, hiccups, common cold, diarrhea, urinary tract infections, urinary frequency, flatulence, priapism, pharyngeal pain, abdmoninal pain, bizarre breathing patterns, burning sensation of tongue, back pain, shoulder pain, chest pain (noncardiac), muscle cramps, paresthesia, increased sweating, falling, syncope, orthostatic hypotension, asthenia (weakness), dysphagia, Horner's syndrome, mydriasis, dry mouth, sialorrhea, neuroleptic malignant syndrome, phlebitis, agranulocytosis, hemolytic and nonhemolytic anemia, rash, gastrointestinal bleeding, duodenal ulcer, Henoch-Schonlein purpura, decreased hemoglobin and hematocrit, thrombocytopenia, leukopenia, angioedema, urticaria, pruritus, alopecia, dark sweat, abnormalities in alkaline phosphatase, abnormalities in SGOT (AST), SGPT (ALT), abnormal Coombs' test, abnormal uric acid, hypokalemia, abnormalities in blood urea nitrogen (BUN), increased creatinine, increased serum LDH, and glycosuria. 


\section{Conclusion}

The Parkinson's disease process is known to be associated with depletion of serotonin, tyrosine hydroxylase, norepinephrine, and dopamine. L-dopa is known to deplete serotonin, serotonin precursors, tyrosine, and the sulfur amino acids. The dosing range of serotonin precursors needed for the individual patient to achieve proper balance with L-dopa administration appears to be in a relatively narrow range with some of the side effects being displayed if the serotonin is either too high or too low.

The most prominent dose-limiting events in the use of L-dopa are the GI symptoms of nausea and vomiting along with psychiatric problems. The patient in this case study had the L-dopa, 5-HTP, L-tyrosine, and L-cysteine administered in proper balance. OCT assay interpretation was implemented early in order to get out in front of amino acid imbalance problems before they occurred.

Everything used in the treatment of this patient is recognized by the FDA as GRAS (generally regarded as safe) and available over the counter without a prescription in the United States.

This paper is the product of nine years of research in the area of serotonin and dopamine precursor administration as guided by OCT assay interpretation and statistical analysis of numerous large databases. The protocol has been refined since 2001 and appears ready for prospective studies. This paper is an attempt to document what is known prior to further studies.

The goal of this paper is to share some of the knowledge gained prior to expanding group studies, spark interest in this area of research, and hold these observations up to scrutiny for Parkinson's disease patients and their caregivers. The administration of properly balanced amino acid precursors used here for Parkinson's disease does hold potential in other research areas as evidenced by previous peer-reviewed writings of the authors since 2009.

\section{Disclosure}

MH discloses ownership of DBS Labs, Duluth, MN, USA. TU discloses directorship of DBS Labs, Duluth, MN, USA. AS reports no conflicts of interest in this work.

\section{References}

1. Barbeau A. L-Dopa therapy in Parkinson's disease: A critical review of nine years' experience. Can Med Assoc J. 1969;101(13): 59-68.

2. Peaston M, Bis-Nchine J. Metabolic studies and clinical observations during L-Dopa treatment of Parkinson's disease. BMJ. 1970;1: $400-403$.
3. FDA-approved carbidopa/levodopa prescribing information. Available from: http://packageinserts.bms.com/pi/pi_sinemet_cr.pdf. Accessed January 20, 2011.

4. FDA-approved ropinirole prescribing information. Available from: http://us.gsk.com/products/assets/us_requip.pdf. Accessed January $20,2011$.

5. FDA-approved amantadine prescribing information. Available from: http:// www.accessdata.fda.gov/drugsatfda_docs/label/2009/016023s041, 018101s016lbl.pdf. Accessed January 20, 2011.

6. FDA-approved trihexylphenidyl prescribing information. Available from: http://www.drugs.com/pro/trihexyphenidyl.html. Accessed January 20, 2011.

7. FDA-approved rasagiline mesylate prescribing information. Available from: http://www.drugs.com/monograph/rasagiline-mesylate.html. Accessed January 20, 2011.

8. FDA-approved entacapone prescribing information. Available from: http://www.pharma.us.novartis.com/product/pi/pdf/comtan.pdf. Accessed January 20, 2011.

9. Mayo Clinic Parkinson's disease. Available from: http://www.mayoclinic. com/health/parkinsons-disease/DS00295/DSECTION=treatmentsand-drugs. Accessed January 20, 2011.

10. Hinz M. Depression. In: Kohlstadt I, editor. Food and Nutrients in Disease Management. Baton Rouge, FL: CRC Press; 2009: 465-481.

11. Hinz M, Stein A, Uncini T. The dual-gate lumen model of renal monoamine transport. Neuropsychiatr Dis Treat. 2010;6:387-392.

12. Hinz M, Stein A, Trachte G, Uncini T. Neurotransmitter testing of the urine, a comprehensive analysis. Open Access Journal of Urology. 2010;2:177-183

13. Hinz M, Stein A, Uncini T. A pilot study differentiating recurrent major depression from bipolar disorder cycling on the depressive pole. Neuropsychiatr Dis Treat. 2010;6:741-747.

14. Stein A, Hinz M, Uncini T. Amino acid responsive Crohn's disease: a case study. Clinical \& Experimental Gastroenterology. 2010;3: $171-177$.

15. Charlton C, Crowell B, Parkinson's disease-like effects of S-adenosylL-methionine: Effects of L-dopa. Pharmacolo Biochem Behav. 1992; 43:423-431.

16. Mones R, Elizan T, Siegel G. Analysis of L-dopa induced dyskenesias in 51 patients with Parkinsonism. J Neurol Neurosurg Psychiatry. 1971;34:668-673.

17. Charlton C. Depletion of nigrostriatal and forebrain tyrosine hydroxylase by S-adenosyl methionine: A model that may explain the occurrence of depression in Parkinson's disease. Life Sci. 1997;61(5) 495-502.

18. Karobath M, Diaz J Huttunen M. The effect of L-dopa on the concentrations of tryptophan, tyrosine, and serotonin in the rat brain. Eur J Pharmacol. 1971;14:393-396.

19. Zhelyaskov D, Levitt M, Udenfriend S. Tryptophan derivatives as inhibitors of tyrosine hydroxylase in vivo and vitro. Mol Pharmacol. $1968 ; 4: 445-451$

20. Garcia N, Berndt T, Tyce G, Knox F. Chronic oral L-DOPA increases dopamine and decreases serotonin excretions. Am J Physiol. 1999;277 (5 Pt 2):R1476-R1480.

21. $\mathrm{Ng} \mathrm{K}$, Chase T, Colburn R, Kopin I. L-Dopa induced release of cerebral monoamines. Science. 1970;170:76-77.

22. Borah A, Kochupurackal P, Mohanakumar P. Long-term L-dopa treatment causes indiscriminate increase in dopamine levels at the cost of serotonin synthesis in discrete brain regions of rats. Cell Mol Neurobiol. 2007;27:985-996.

23. Soares-da-Silva P, Pinto-do-O P. Antagonistic actions of renal dopamine and 5-hydroxytryptamine: effects of amine precursors on the cell inward transfer and decarboxylation Br J Pharmacol. 1996;117: 1187-1192.

24. Wuerthele S, Moore K. Studies of the mechanisms of L-dopa induced depletion of 5-hydroxytryptamine in the mouse brain. Life Sci. 1977; 20:1675-1680. 
25. Zeevalk G, Manzino L, Sonsalla PK, Bernard LP. Characterization of intracellular elevation of glutathione ( $\mathrm{GSH}$ ) with glutathione monoethyl ester and GSH in brain and neuronal cultures: Relevance to Parkinson's disease. Exp Neurol. 2007;203:512-520.

26. Ritvo E, Yuwiler A, Geller E. Effects of L-dopa in autism. JAutism Dev Disord. 1971;1(2).

27. Benson R, Crowell B, Hill B. The effects of L-Dopa on the activity of methionine adenosyltransferase: Relevance to L-Dopa therapy and tolerance. Neurochemical Res.1993;18(3):325-330.

28. Liu X, Wilson K, Charlton C. Effects of L-dopa treatment on methylation in mouse brain: Implications for side effects of L-dopa. Life Sci. 2000;66(23):2277-2288.

29. Fuller R, Hemrick-Luecke S, Perry K. Effects of L-dopa on epinephrine concentration in rat brain: Possible role of inhibition of norepinephrine $\mathrm{N}$-methyl transferase by S-adenosyl homocysteine. J Pharmacol Exp Ther. 1982;223(1):84-89.

30. FDA-approved carbidopa/L-dopa prescribing information. Available from: http://packageinserts.bms.com/pi/pi_sinemet_cr.pdf. Accessed January 19, 2011.

31. Katzenschlager R, Evans A, Manson A, et al. Mucuna pruriens in Parkinson's disease: A double blind clinical and pharmacological study. J Neurol Neurosurg Psychiatry. 2004;75:1672-1677.
32. Trachte G, Uncini T, Hinz M. Both stimulatory and inhibitory effects of dietary 5-hydroxytryptophan and tyrosine are found on urinary excretion of serotonin and dopamine in a large human population. Neuropsychiatr Dis Treat. 2009;5:227-235.

33. Unified Parkinson's disease rating scale (UPDRS). Available from: http://www.mdvu.org/library/ratingscales/pd/updrs.pdf. Accessed January 19, 2011.

34. Menza M, Marin H, Kaufman K. Citalopram treatment of depression in Parkinson's disease: The impact on anxiety, disability, and cognition. J Neuropsychiatry Clin Neurosci. 2004;16(3):315-319.

35. Andrews D, Patrick R, Barchas J. The effects of 5-hydroxytryptophan and 5-hydroxytryptamine on dopamine synthesis and release in rat brain striatal synaptosomes. J Neurochem. 1978;30:465-470.

36. Awazi N. Guldberg H. On the interaction of 5-hydroxytryptophan and 5-hydroxytryptamine with dopamine metabolism in the rat striatum arch. Pharmacology. 1978;303:63-72.

37. Trachte G, Uncini T, Hinz M. Both stimulatory and inhibitory effects of dietary 5-hydroxytryptophan and tyrosine are found on urinary excretion of serotonin and dopamine in a large human population. Neuropsychiatr Dis Treat. 2009;5:227-235.

38. Koepsell H, Schmitt B, Gorboulev V. Organic cation transporters. Physiol Biochem Pharmacol. 2003;150:36-90.
International Journal of General Medicine

\section{Publish your work in this journal}

The International Journal of General Medicine is an international, peer-reviewed open-access journal that focuses on general and internal medicine, pathogenesis, epidemiology, diagnosis, monitoring and treatment protocols. The journal is characterized by the rapid reporting of reviews, original research and clinical studies across all disease areas.

\section{Dovepress}

A key focus is the elucidation of disease processes and management protocols resulting in improved outcomes for the patient. The manuscript management system is completely online and includes a very quick and fair peer-review system. Visit http://www.dovepress.com/ testimonials.php to read real quotes from published authors. 